\title{
Design and Simulation of Rectangular Micro-strip Dual Band Single Slot Patch Antenna
}

\author{
Abhinav BhargavaPh.D. \\ Scholar, Dept. of ECE \\ BUIT, Bhopal(M.P.) \\ India
}

\author{
Poonam Sinha,Ph.D \\ Dept. of ECE \\ BUIT, Bhopal(M.P.) \\ India
}

\begin{abstract}
The design of micro strip antenna is vital study for today's wireless communication system to achieve compatibility. Research and development in micro strip antenna, exploiting their advantages such as low weight, low volume, and low cost, conformal configuration compatibility with integrated circuits. This paper presents a proposed design for Rectangular micro-strip patch antenna by cutting a rectangular slot of $(2 * 30)$ in the Rectangular patch which operates at two central frequencies $1.4,2.37 \mathrm{GHz}$. Field generated by this slot should be additive in the nature so that we are able to radiate electromagnetic radiation properly, which is a new dual frequency micro strip antenna. Basically micro strip patch is having small bandwidth and can be operated at single frequency. Return loss, VSWR and bandwidth has been found by different micro strip feeding technique. All available feeding technique are micro strip, aperture coupled, proximity feed etc. For the design of the micro strip antenna FR-4 substrates which have permittivity of 4.4 and thickness 3.2, loss tangent is 0.02 has been used. We are using FEKO simulation software for designing and analysis. We have observed that using slotted patch antenna and using micro strip at proper location we can get better return loss, VSWR bandwidth and multiband.
\end{abstract}

\section{General Terms}

Antenna designing, Microwave communication, Radiation Pattern.

\section{Keywords}

Slotted Rectangular micro-strip patch antenna, return loss, VSWR.

\section{INTRODUCTION}

Modern telecommunication device are require to be small and able to integrate several functionalities. The antenna used for these wireless systems must hence possess multiband capabilities but yet remain compact. Antenna is a key building in wireless communication and global positioning system(GPS) since it was first demonstrate in 1886 by Heinrich Hertz and its practical application by Guglielmo Marconi in 1901[1]. Future trend in communication design is towards compact devices. Low cost of fabrication and low profile features attract many researches to investigate the performance of a micro-strip patch antenna in various ways. Micro-strip antenna was first proposed by G.A. Deschamps in 1953. Micro-strip patch antennas are often uses where thickness and conformability to the host surfaces are the key requirements. Since patch antennas can be directly printed onto a circuit board, these are becoming increasingly popular within the mobile phone market. They are low cost, have a low profile and are easily fabricated. One of the key drawbacks of such device is their narrow bandwidth. Microstrip patch antenna is widely considered to be suitable for many wireless applications, even though it usually has a narrow bandwidth.

The bandwidth limitation can be addressed by using thick substrates, cutting slots in the metallic patch, using aperture coupled stacked patch antenna. The stacked patch antenna has multilayer structure consisting of several parasitic radiating elements placed one above the other and above the driven element. However this approach has the inherent disadvantage of increased overall thickness and issues related on aligning various precisely. In this paper we design a rectangular microstrip patch antenna in which rectangular shaped slots are cut. By cutting a slot in micro-strip patch enhance its bandwidth. For wireless communication systems, the antenna is one of the most critical components. A good design of the antenna can relax system requirements and improve overall system performance. A typical example is TV for which the overall broadcast reception can be improved by utilizing a highperformance antenna. The antenna serves to a communication system the same purpose that eyes and eyeglasses serve to a human.[1] The individual antenna elements in an array are fed using a feed network. The complexity of the feed network depends on the number of elements, the amplitude and/or phase distribution between the elements, the ability to do beam steering. It is important to realize that the feed network is the most complex part of the array. [1]

Some reflector antennas employ a parabolic cylinder as the reflecting surface while a more common reflecting surface shape is the paraboloid (parabolic dish antenna). The so-called Cassegrain antenna uses dual reflecting surfaces (the main reflector is a paraboloid, the sub-reflector is a hyperboloid). [2] Lenses are primarily used to collimate incident divergent energy to prevent it from spreading in undesired directions. By properly shaping the geometrical configuration and choosing the appropriate material of the lenses, they can transform various forms of divergent energy into plane waves. They can be used in most of the same applications as are the parabolic reflectors, especially at higher frequencies. Their dimensions and weight become exceedingly large at lower frequencies. Lens antennas are classified according to the material from which they are constructed, or according to their geometrical shape. [2]

\section{ANTENNA DESIGN}

The proposed antenna design by cutting rectangular slots in Rectangular patch as shown in fig. (1). Cutting of these slots 
in antenna increases the current path which increases current intensity as a result efficiency is increased. First a rectangular micro-strip patch antenna is designed based on standard design procedure to determine the length (L) and width (W) for resonant frequency. The resonant frequency of micro-strip antenna and the size of the radiation patch can be similar to the following formulas

$f=\frac{C}{2 L \sqrt{\varepsilon}}$

$\mathrm{W}=\frac{c}{2 f} \sqrt{\frac{2}{\varepsilon+1}}$

$\mathrm{L}=\frac{C}{2 f \sqrt{\varepsilon}}-2 \Delta \mathrm{L}$

Where $f$ is the resonant frequency of the antenna, $\mathrm{C}$ is the free space velocity of the light, $\mathrm{L}$ is the actual length of the current, $\varepsilon$ is the effective dielectric constant of the substrate and $\Delta \mathrm{L}$ is the length of equivalent radiation gap. The dimension of the patch are $\mathrm{L}=50 \mathrm{~mm}$ and $\mathrm{W}=60 \mathrm{~mm}$. Inside this rectangular patch rectangular shaped slots are cut. The antenna is fabricated on a substrate of dielectric constant 4.4 and thickness $\mathrm{h}=3.2 \mathrm{~mm}$. The microstrip feeding is used for optimum results. The microstrip feeding have advantages as well as disadvantages over the coaxial or probe feeding technique, like gain can be increases by some of the percentage as compare to microstrip feeding, probe feeding is quite easy to apply on the antenna.

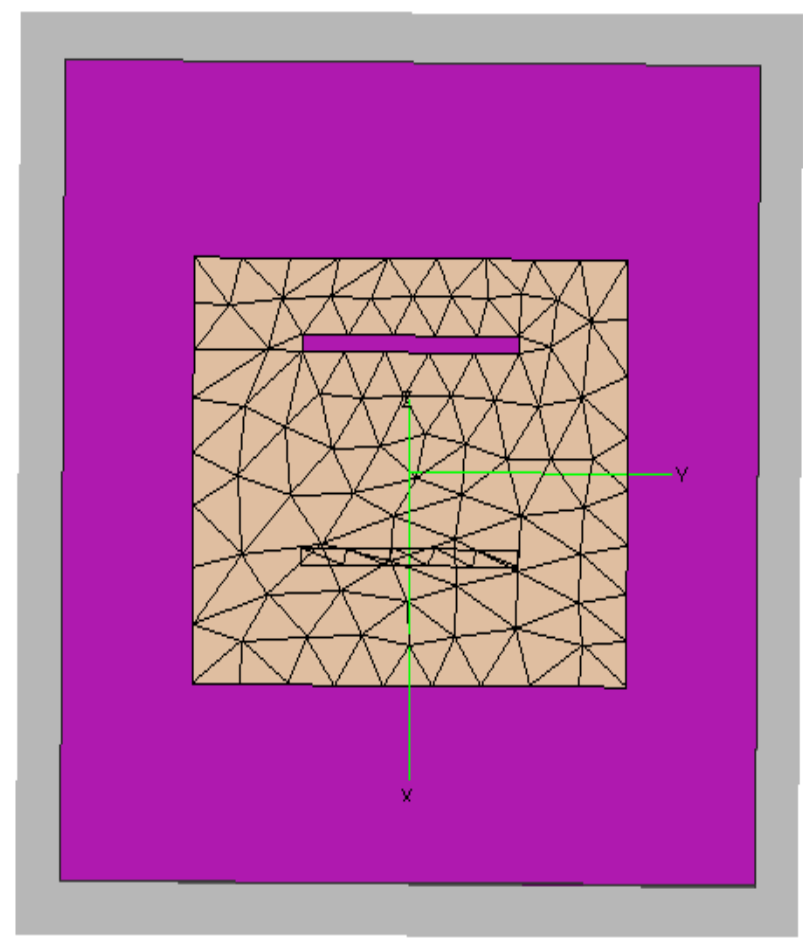

Fig 1 : Proposed Rectangular Micro-strip Patch Antenna with single slot.

\section{ANTENNA RESULT}

The simulation of micro-strip patch antenna is done by using FEKO simulation software. This software is very useful for patch antenna designing because of its user compatibility and availability of graphics tool and cutting tool. The variation of return loss with frequency of rectangular patch antenna with a rectangular slots shown in figure (2). The return loss is defined as the ratio of the Fourier transform of the incident pulse and the reflected signal. It is an important parameter. The VSWR is defined as the ratio of incident wave and reflected wave, which is important to improve the system performance. The VSWR graph for a rectangular slotted rectangular patch antenna is shown in figure (3)

The VSWR indicates the mismatch between the antenna and the transmission line. For perfect matching the VSWR value should be close to unity. The simulation impedance bandwidth for the rectangular slot loaded rectangular microstrip patch antenna as shown in fig. (1) is $100 \mathrm{MHz}$ and it is about $4.5 \%$ and the best return loss $\left(\mathrm{S}_{11}\right)$ is $-17 \mathrm{~dB}$ at $1.4 \mathrm{GHz}$ and $24 \mathrm{~dB}$ at $2.37 \mathrm{GHz}$. The bandwidth is calculated at the frequency range where the return loss is approximately below the $-10 \mathrm{~dB}$. The simulated radiation pattern in $2 \mathrm{D}$ are shown in figure (4) and the Smith chart is shown in figure (5) and radiation pattern in 3D is also shown in figure (6) for the rectangular Slotted rectangular micro-strip patch antenna.

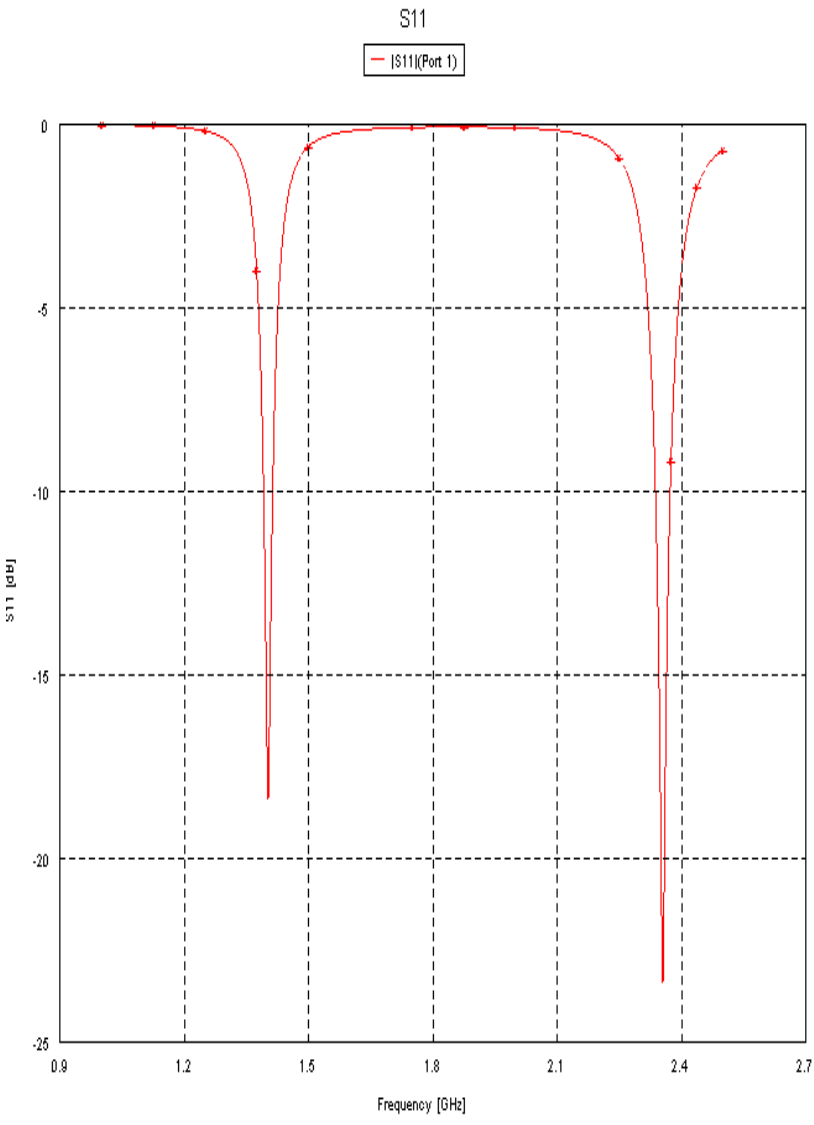

Fig 2 : Return loss of the Proposed Rectangular Microstrip Patch Antenna with single slots 


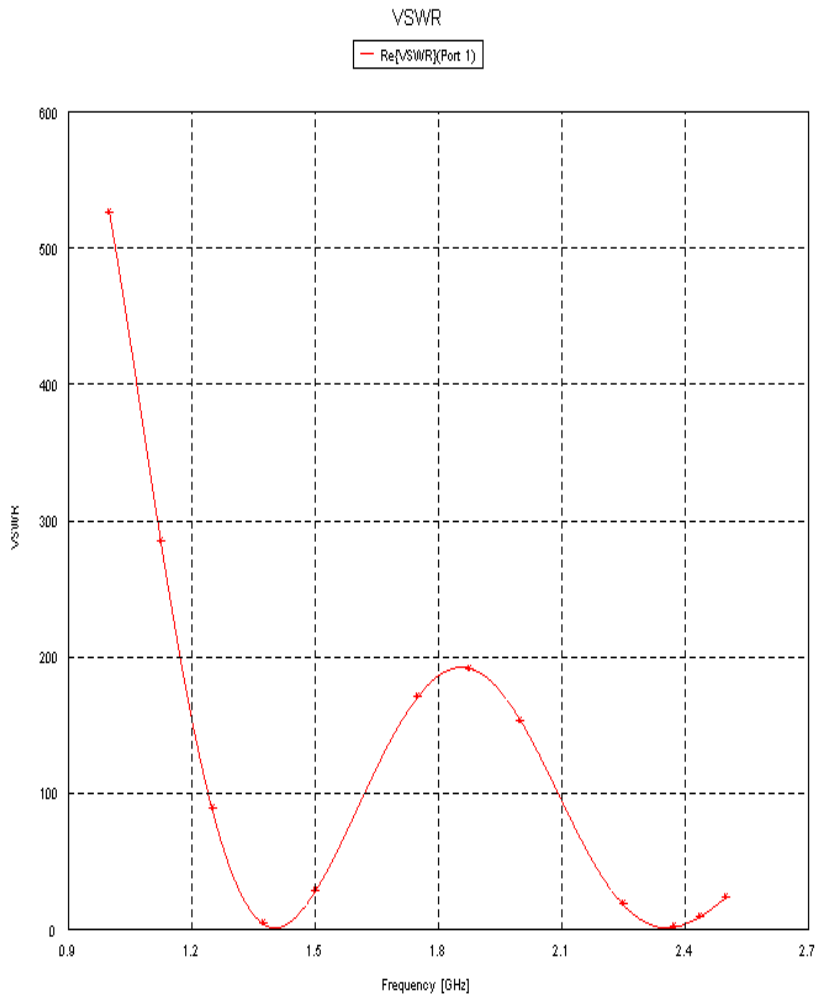

Fig 3 : VSWR of the Proposed Rectangular Micro-strip Patch Antenna with single slot

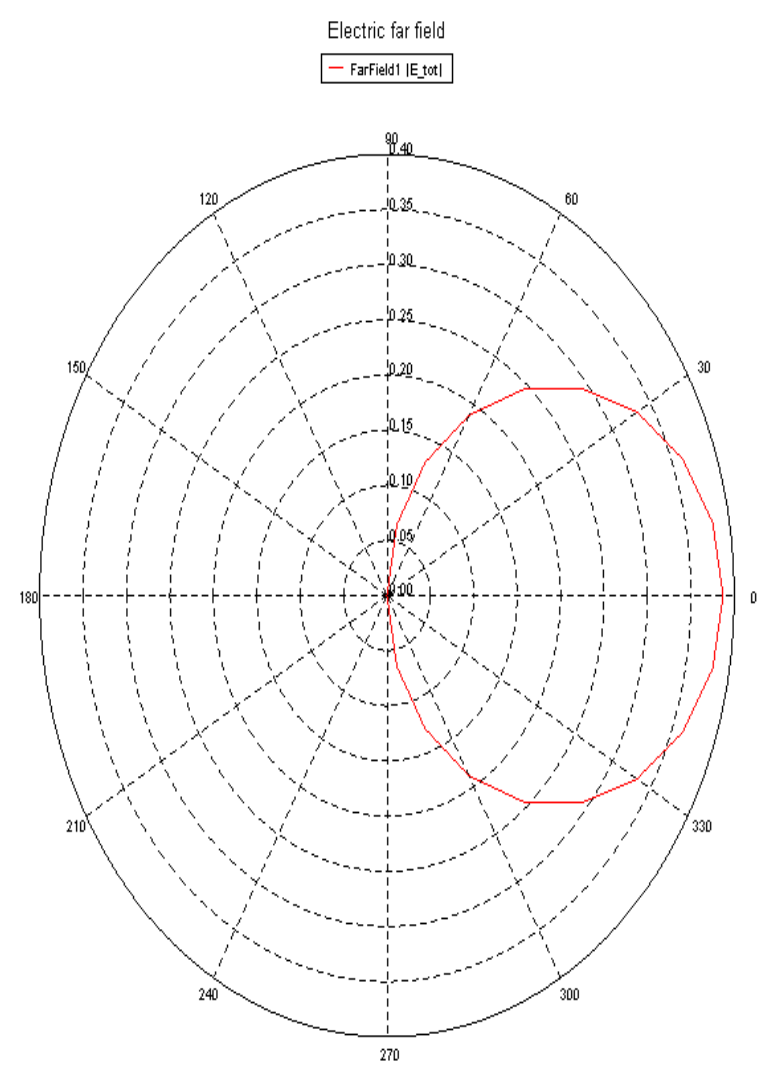

Fig 4: Radiation pattern in 2D of the Proposed Rectangular Micro-strip Patch Antenna with single slot

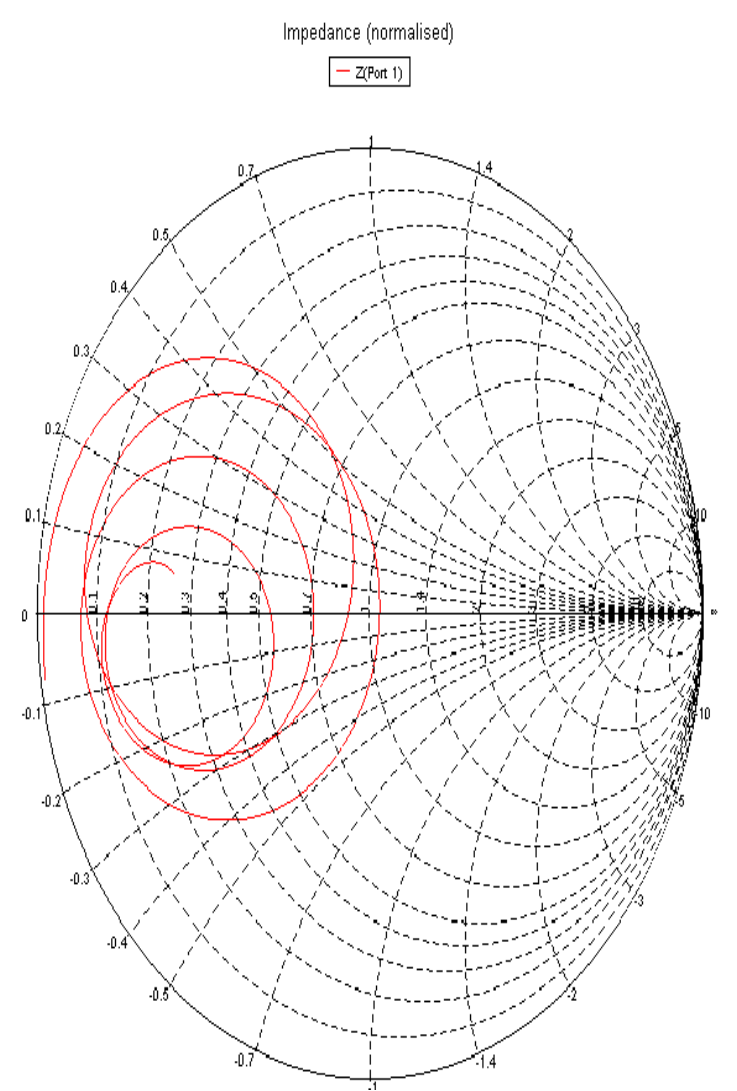

Fig 5: Smith chart of the Proposed Rectangular Microstrip Patch Antenna with rectangular slots

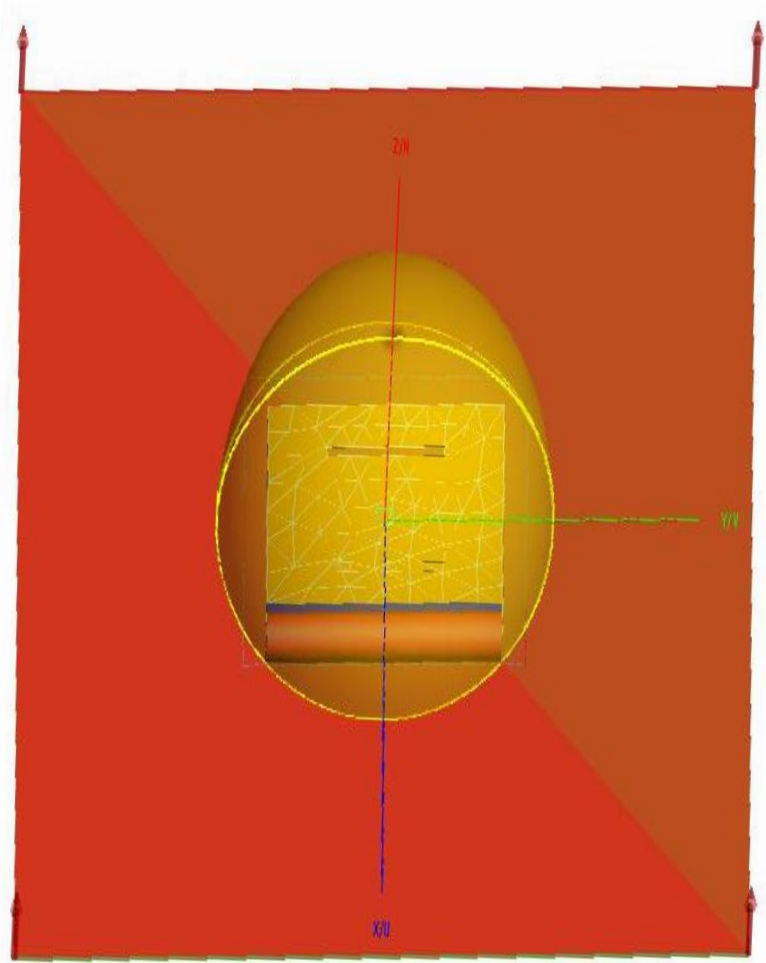

Fig 6: Radiation pattern in 3D of the Proposed Rectangular Micro-strip Patch Antenna with single slot 


\section{CONCLUTION}

The proposed slotted microstrip antenna is small and suitable to handle easily. It is observed that a microstrip feed, rectangular slotted rectangular micro-strip patch antenna with multiband is presented. The proposed antenna has a compact size of $(50 * 60)$ and it can effectively covers the Wi-Max application and other wireless applications (cellular mobile communication) because of multiband capabilities. In the investigation the bandwidth of $23 \%$ has been achieved.

\section{REFERENCES}

[1] Constantine A. Balanis "Antenna Theory: Analysis and Design", Third Edition A John Wiley \& sons, inc., publication. PP 232-437, Edition 2005.

[2] K. D. Prasad and Manish Prasad "Introduction to Antenna and Wave Propagation". PP 529-599, 772-880, Edition 2003.
[3] G.Papitha, V. Thyagarajan and AyshathulFouzia,'Design and Simulation of Stacked Fractal Patch Antenna using IE3D"

[4] John D Kraus, Ronald J Marhefka, Ahmed S Khan," Antennas and Wave Propagation". Tata McGraw Hill Education Private Limited New Delhi 2010.

[5] R. Garg and P. Bhartia, "Micro-strip Antenna handbook". Boston Artech House 2001.

[6] R.Q. Lee, K.F. Lee and J.Bobinchak,"Characterstics of a two layer electromagnetically coupled rectangular patch antenna", Electron Lett. Vol. 23 no 20, pp 1070-1072, 1987

[7] Binu Paul, S. Mridula, C.K. Aanandan and P. Mohan. Microwave and optical technology letters/ vol33, no4, 4 may2002. 